\title{
Caractérisation spectroscopique et étude structurale de $\operatorname{RbEr}\left(\mathrm{PO}_{3}\right)_{4}$
}

\author{
K. Horchani ${ }^{1}$, J. Amami ${ }^{1}$, D. Merle ${ }^{2}$ et M. Férid ${ }^{1}$ \\ 1 Laboratoire des Procédés Chimiques, Institut National de Recherche Scientifique et \\ Technique, BP. 95, 2050 Hammam-Lif, Tunisie \\ 2 Centre de Diffractométrie UCB Lyon 1, Bâtiment Jules Raulin, 43 Bd. 11 novembre 1918, \\ 69622 Villeurbanne, France
}

\begin{abstract}
Crystals of $\operatorname{RbEr}\left(\mathrm{PO}_{3}\right)_{4}$ have been grown by the flux technique and characterized by X-ray diffraction, UV-Vis absorbance and infrared spectroscopy. Single crystal structure of $\operatorname{RbEr}\left(\mathrm{PO}_{3}\right)_{4}$ has been solved, it crystallizes in the monoclinic $\mathrm{P} 2{ }_{1} / \mathrm{n}$ space group with lattice parameters : $a=10,2419$ (2), $b=8,8349(2), c=10,9361(2) \AA, B=106,229(2)^{\circ}, Z=4$. The crystal structure has been refined yielding a final $\mathrm{R}\left(\mathrm{F}^{2}\right)=0.026$ for 2206 independent reflections $\left(\mathrm{F}_{\mathrm{o}}{ }^{2} \geq 2 \sigma\left(\mathrm{F}_{\mathrm{o}}{ }^{2}\right)\right)$. The $\operatorname{RbEr}\left(\mathrm{PO}_{3}\right)_{4}$ structure is characterized by helical ribbons $\left(\mathrm{PO}_{3}\right)_{\infty}$ running along the c-axis. The erbium ions have an eightfold coordination. The $\mathrm{ErO}_{8}$ polyhedra are isolated from each other in the sense that they do not share any oxygen atom. The minimal distance between two atoms of erbium is $6,2583 \AA$.
\end{abstract}

\begin{abstract}
Résumé. Le polyphosphate $\mathrm{RbEr}\left(\mathrm{PO}_{3}\right)_{4}$ est synthétisé, sous forme monocristalline, par la méthode de flux ; ce composé est caractérisé par diffraction RX, spectroscopie d'absorption optique et spectroscopie de vibration IR. L'étude structurale de $\operatorname{RbEr}\left(\mathrm{PO}_{3}\right)_{4}$ a montré que ce polyphosphate cristallise dans le système monoclinique, de groupe d'espace $\mathrm{P} 2{ }_{1} / \mathrm{n}$, les paramètres de maille sont : $a=10,2419(2), b=$ $8,8349(2), c=10,9361(2) \AA, \beta=106,229(2)^{\circ}, Z=4$. L'affinement conduit à un facteur de confiance $\mathrm{R}\left(\mathrm{F}^{2}\right)=0.026$, pour 2206 réflexions indépendantes $\left(\mathrm{F}_{\mathrm{o}}{ }^{2} \geq 2 \sigma\left(\mathrm{F}_{\mathrm{o}}{ }^{2}\right)\right)$. La structure de $\operatorname{RbEr}\left(\mathrm{PO}_{3}\right)_{4}$ est caractérisée par une chaîne hélicoïdale $\left(\mathrm{PO}_{3}\right)_{\infty}$ se développant le long de l'axe c. Les atomes d'erbium ont un environnement de 8 oxygènes. Les polyèdres $\mathrm{ErO}_{8}$ sont isolés les uns des autres, ils ne présentent aucun atome d'oxygène en commun. La distance Er-Er la plus courte mesure 6,258 (§).
\end{abstract}

\section{INTRODUCTION}

De nombreux travaux de recherche ont montré que les matériaux phosphatés à base de terres rares peuvent présenter des propriétés optiques intéressantes [1-3]. Ces propriétés peuvent être optimisées grâce à la grande variété structurale de ces phosphates. Récemment des recherches sur des matériaux phosphatés codopé $\mathrm{Yb}^{3+}-\mathrm{Er}^{3+}$ ont montré que ces composés sont prometteurs pour la réalisation de lasers à sécurité oculaire utilisés dans le domaine de la télémétrie et la détection de polluants dans l'atmosphère [4]. Dans ce travail nous présentons la synthèse et la caractérisation du polyphosphate d'erbium $\operatorname{RbEr}\left(\mathrm{PO}_{3}\right)_{4}$. 


\section{PARTIE EXPÉRIMENTALE}

Le polyphosphate $\operatorname{RbEr}\left(\mathrm{PO}_{3}\right)_{4}$ est synthétisé, sous forme monocristalline, par la méthode de flux, les cristaux sont obtenus à partir de carbonate de rubidium $\mathrm{Rb}_{2} \mathrm{CO}_{3}$, d'oxyde d'erbium $\mathrm{Er}_{2} \mathrm{O}_{3}$ et d'acide phosphorique $\mathrm{H}_{3} \mathrm{PO}_{4}$. Le mélange est chauffé à une température de $300^{\circ} \mathrm{C}$ dans un creuset en carbone vitreux et maintenu à cette température pendant une semaine.

Le spectre d'absorption optique des cristaux a été enregistré de 200 à $1100 \mathrm{~nm}$, à l'aide d'un spectrophotomètre Perkin-Elmer, UV-VIS NIR Lambda 20.

Le spectre d'absorption infrarouge a été enregistré à l'aide d'un spectromètre FTIR 2000 (PerkinElmer), sur un échantillon pastillé dans du KBr à l'aide d'une presse.

La collecte des intensités a été effectuée à l'aide d'un diffractomètre automatique à quatre cercles de type Nonius Kappa CCD utilisant la radiation $K_{\alpha}$ du molybdène $(\lambda=0,71073 \AA)$. La structure a été résolue, par exploitation de la fonction tridimensionnelle de Patterson au moyen du programme WinGX [5] utilisant SHELXS 97 [6] puis affinée par SHELXL 97 [7].

\section{RÉSULTATS ET DISCUSSION}

Le polyphosphate $\operatorname{RbEr}\left(\mathrm{PO}_{3}\right)_{4}$ cristallise dans le système monoclinique, de groupe d'espace $\mathrm{P} 2 / 1 \mathrm{n}$, les paramètres de maille sont : $a=10,2419(2), b=8,8349(2), c=10,9361(2) \AA ̊ ., B=106,229(2)^{\circ}, Z=4$. L'affinement conduit à un facteur de confiance de 2,6\%, pour 2206 réflexions indépendantes. $\operatorname{RbEr}\left(\mathrm{PO}_{3}\right)_{4}$ est isotype au composé $\operatorname{TlNd}\left(\mathrm{PO}_{3}\right)_{4}$ [8]. Les conditions de la collecte ainsi que les principaux résultats de l'affinement sont consignés dans le tableau 1. Le tableau 2 donne les principales distances interatomiques dans la structure de $\operatorname{RbEr}\left(\mathrm{PO}_{3}\right)_{4}$.

Tableau 1. Données cristallographiques, conditions de la collecte et résultats de l'affinement de la structure de $\operatorname{RbEr}\left(\mathrm{PO}_{3}\right)_{4}$

\begin{tabular}{|c|c|}
\hline \multicolumn{2}{|l|}{ DONNEES CRISTALLOGRAPHIQUES } \\
\hline Formule chimique : & $\operatorname{RbEr}\left(\mathrm{PO}_{3}\right)_{4}$ \\
\hline Système cristallin : monoclinique & $a=10,2419(2) \AA, b=8,8349(2) \AA$ \\
\hline \multirow[t]{2}{*}{ Groupe d'espace $: \mathrm{P} 2_{1} / \mathrm{n}$} & $\mathrm{c}=10,9361(2) \AA, \beta=106,229(2)^{\circ}$ \\
\hline & $V=950,13 \AA^{3}, Z=4$ \\
\hline $\mathrm{F}(000)=$ & 1044 \\
\hline$\rho_{\text {cal }}\left(\mathrm{g} \cdot \mathrm{cm}^{-3}\right)$ & 3,975 \\
\hline Masse molaire $\left(\mathrm{g} \cdot \mathrm{mol}^{-1}\right)$ & 568,6 \\
\hline Coefficient d'absorption $\left(\mathrm{mm}^{-1}\right)$ & 14,66 \\
\hline Dimensions du cristal $\left(\mathrm{mm}^{3}\right)$ & $0,16 \times 0,15 \times 0,2$ \\
\hline Domaine angulaire $\left(^{\circ}\right)$ : & $2,34<\theta<57,41$ \\
\hline Domaine de mesure : & $-13<\mathrm{h}<13,-10<\mathrm{k}<11,-14<1<14$ \\
\hline Nombre de réflexions indépendantes : & 2441 \\
\hline Correction d'absorption : & Intégration \\
\hline Réflexions uniques incluses: & 2206 pour $\mathrm{I}>2 \sigma(\mathrm{I})$ \\
\hline Schéma de pondération WGHT = & $1 /\left[\sigma^{2}\left(\mathrm{~F}_{\mathrm{o}}^{2}\right)+(0,1000 \mathrm{P})^{2}\right.$ où $\mathrm{P}=\left(\mathrm{F}_{\mathrm{o}}^{2}+2 \mathrm{~F}_{\mathrm{c}}^{2}\right) / 3$ \\
\hline Nombre de paramètres affinés & 164 \\
\hline Facteurs de reliabilité & $\mathrm{R}=0,026, \mathrm{Rw}=0,0956$ \\
\hline
\end{tabular}


Tableau 2. Principales distances inter atomiques dans la structure de $\operatorname{RbEr}\left(\mathrm{PO}_{3}\right)_{4}$.

\begin{tabular}{|c|c|c|c|c|c|c|c|}
\hline \multicolumn{4}{|c|}{ Tétraèdres $\mathrm{PO}_{4}$} & \multirow{2}{*}{\multicolumn{4}{|c|}{ Tétraèdre autour de $\mathrm{P}_{2}$}} \\
\hline \multicolumn{4}{|c|}{ Tétraèdre autour de $\mathrm{P}_{1}$} & & & & \\
\hline $\mathrm{P}_{1}-\mathrm{O}_{4}$ & $1,604(3)$ & $\mathrm{O}_{4}-\mathrm{P}_{1}-\mathrm{O}_{5}$ & $111,1(2)$ & $\mathrm{P}_{2}-\mathrm{O}_{2}$ & $1,474(3)$ & $\mathrm{O}_{2}-\mathrm{P}_{2}-\mathrm{O}_{11}$ & $118,6(2)$ \\
\hline $\mathrm{P}_{1}-\mathrm{O}_{5}$ & $1,493(3)$ & $\mathrm{O}_{4}-\mathrm{P}_{1}-\mathrm{O}_{3}$ & $102,1(2)$ & $\mathrm{P}_{2}-\mathrm{O}_{7}$ & $1,599(3)$ & $\mathrm{O}_{2}-\mathrm{P}_{2}-\mathrm{O}_{12}$ & $109,3(2)$ \\
\hline $\mathrm{P}_{1}-\mathrm{O}_{3}$ & $1,604(3)$ & $\mathrm{O}_{3}-\mathrm{P}_{1}-\mathrm{O}_{5}$ & $108,9(2)$ & $\mathrm{P}_{2}-\mathrm{O}_{11}$ & $1,483(3)$ & $\mathrm{O}_{2}-\mathrm{P}_{2}-\mathrm{O}_{7}$ & $108,1(2)$ \\
\hline \multirow[t]{3}{*}{$\mathrm{P}_{1}-\mathrm{O}_{10}$} & $1,481(3)$ & $\mathrm{O}_{3}-\mathrm{P}_{1}-\mathrm{O}_{10}$ & $108,8(2)$ & $\mathrm{P}_{2}-\mathrm{O}_{12}$ & $1,589(3)$ & $\mathrm{O}_{11}-\mathrm{P}_{2}-\mathrm{O}_{12}$ & $109,9(2)$ \\
\hline & & $\mathrm{O}_{4}-\mathrm{P}_{1^{-}}-\mathrm{O}_{10}$ & $107,5(2)$ & & & $\mathrm{O}_{11}-\mathrm{P}_{2}-\mathrm{O}_{7}$ & $110,4(2)$ \\
\hline & & $\mathrm{O}_{5}-\mathrm{P}_{1}-\mathrm{O}_{10}$ & $117.3(2)$ & & & $\mathrm{O}_{12}-\mathrm{P}_{2^{-}} \mathrm{O}_{7}$ & $98,5(2)$ \\
\hline \multicolumn{8}{|c|}{ Tétraèdre autour de $\mathrm{P}_{3}$} \\
\hline $\mathrm{P}_{3}-\mathrm{O}_{6}$ & $1,485(3)$ & $\mathrm{O}_{6}-\mathrm{P}_{3}-\mathrm{O}_{9}$ & $118,5(2)$ & \multicolumn{4}{|c|}{ Tétraèdre autour de $\mathrm{P}_{4}$} \\
\hline $\mathrm{P}_{3}-\mathrm{O}_{4}$ & $1,605(3)$ & $\mathrm{O}_{6}-\mathrm{P}_{3}-\mathrm{O}_{12}$ & $111,0(2)$ & $\mathrm{P}_{4}-\mathrm{O}_{8}$ & $1,471(4)$ & $\mathrm{O}_{8}-\mathrm{P}_{4^{-}}-\mathrm{O}_{1}$ & $121,2(2)$ \\
\hline $\mathrm{P}_{3}-\mathrm{O}_{9}$ & $1,484(3)$ & $\mathrm{O}_{6}-\mathrm{P}_{3}-\mathrm{O}_{4}$ & $109,7(2)$ & $\mathrm{P}_{4}-\mathrm{O}_{1}$ & $1,487(4)$ & $\mathrm{O}_{8}-\mathrm{P}_{4^{-}}-\mathrm{O}_{7}$ & $106,9(2)$ \\
\hline $\mathrm{P}_{3}-\mathrm{O}_{12}$ & $1,605(3)$ & $\mathrm{O}_{9}-\mathrm{P}_{3}-\mathrm{O}_{12}$ & $109,7(2)$ & $\mathrm{P}_{4}-\mathrm{O}_{7}$ & $1,604(3)$ & $\mathrm{O}_{8}-\mathrm{P}_{4^{-}} \mathrm{O}_{3}$ & $111,7(2)$ \\
\hline & & $\mathrm{O}_{9}-\mathrm{P}_{3}-\mathrm{O}_{4}$ & $107,5(2)$ & $\mathrm{P}_{4}-\mathrm{O}_{3}$ & $1,614(3)$ & $\mathrm{O}_{1}-\mathrm{P}_{4^{-}} \mathrm{O}_{7}$ & $110,1(2)$ \\
\hline & & $\mathrm{O}_{12}-\mathrm{P}_{3}-\mathrm{O}_{4}$ & $98,4(2)$ & & & $\mathrm{O}_{1}-\mathrm{P}_{4^{-}} \mathrm{O}_{3}$ & $106,1(2)$ \\
\hline \multicolumn{4}{|c|}{ Polyèdre $\mathrm{ErO}_{8}$} & & & $\mathrm{O}_{7}-\mathrm{P}_{4^{-}} \mathrm{O}_{3}$ & $98,5(2)$ \\
\hline $\mathrm{Er}-\mathrm{O}_{8}$ & $2,327(3)$ & $\mathrm{Er}-\mathrm{O}_{9}$ & $2,268(3)$ & & & & \\
\hline $\mathrm{Er}-\mathrm{O}_{11}$ & $2,306(3)$ & $\mathrm{Er}-\mathrm{O}_{2}$ & $2,360(3)$ & & & & \\
\hline $\mathrm{Er}-\mathrm{O}_{10}$ & $2,362(3)$ & $\mathrm{Er}-\mathrm{O}_{6}$ & $2,386(3)$ & & & & \\
\hline $\mathrm{Er}-\mathrm{O}_{1}$ & $2,346(3)$ & $\mathrm{Er}-\mathrm{O}_{5}$ & 2,417 (3) & & & & \\
\hline
\end{tabular}

La structure de $\mathrm{RbEr}\left(\mathrm{PO}_{3}\right)_{4}$ est caractérisée par une chaîne hélicoïdale $\left(\mathrm{PO}_{3}\right)_{\infty}$ se développant le long de l'axe c. La projection de l'arrangement atomique sur le plan (100), (fig.1) montre que les tétraèdres $\mathrm{PO}_{4}$ sont liés par l'intermédiaire d'atomes d'oxygènes pour former des chaînes.

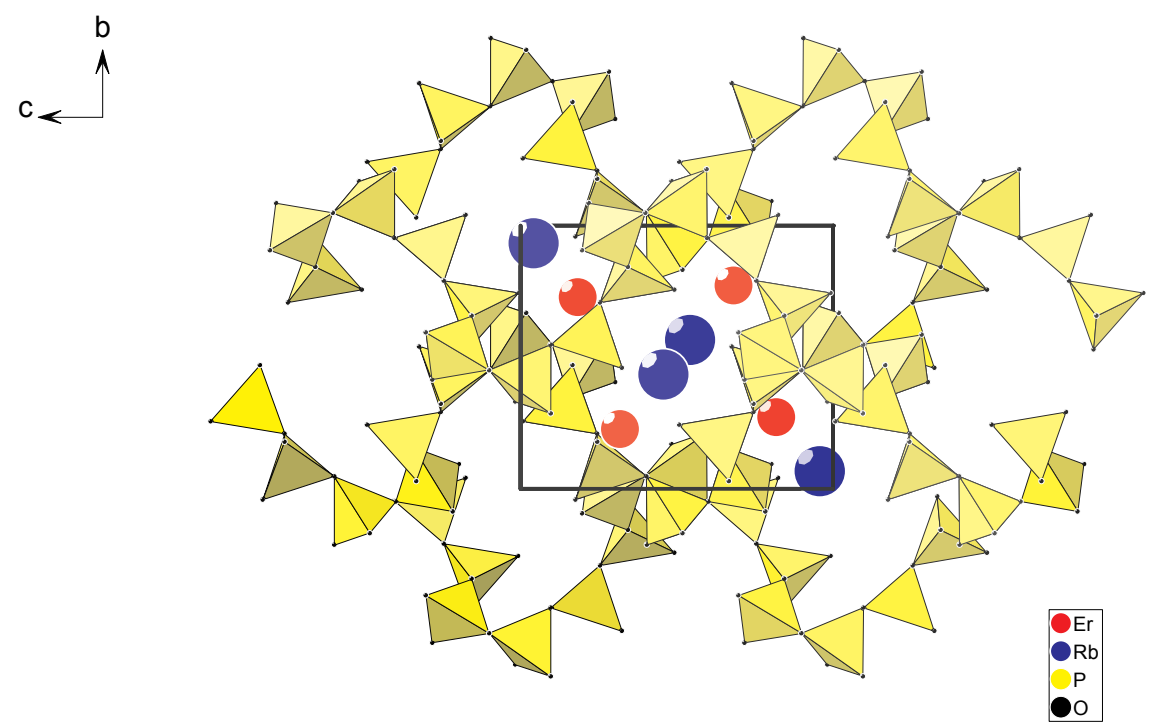

Figure 1. Projection de la structure de $\operatorname{RbEr}\left(\mathrm{PO}_{3}\right)_{4}$ dans le plan (100). 
L'environnement le plus proche du cation $\mathrm{Er}^{3+}$ est constitué de 8 atomes d'oxygène. Les polyèdres $\mathrm{ErO}_{8}$ sont des antiprismes isolés les uns des autres de forme géométrique légèrement déformée (fig.2 ) et ils ne présentent aucun atome d'oxygène en commun. La distance Er-Er la plus courte mesure $6,258 \AA$.

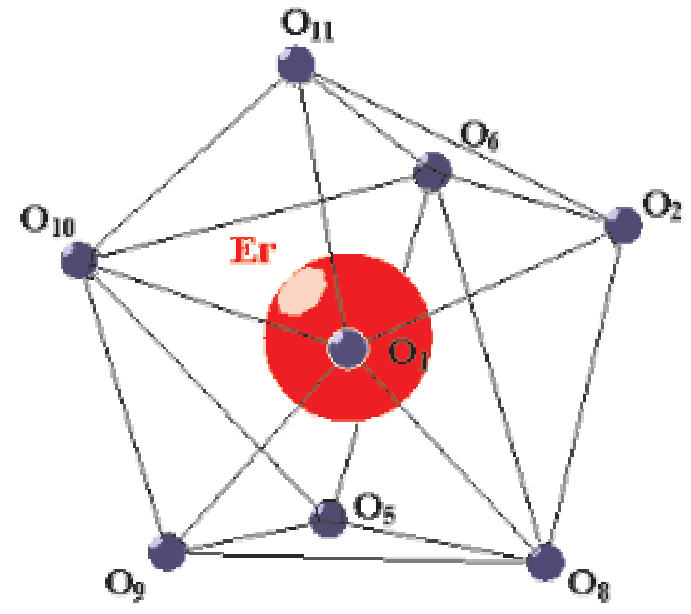

Figure 2. Environnement de l'erbium dans $\mathrm{RbEr}\left(\mathrm{PO}_{3}\right)_{4}$.

Le spectre d'absorption optique est enregistré à la température ambiante dans le domaine 200-1100 nm (fig.3); les transitions du niveau fondamental ${ }^{4} \mathrm{I}_{15 / 2}$ vers les niveaux excités ${ }^{4} \mathrm{I}_{11 / 2},{ }^{4} \mathrm{I}_{9 / 2},{ }^{4} \mathrm{~F}_{9 / 2},{ }^{4} \mathrm{~S}_{3 / 2}$, ${ }^{2} \mathrm{H}_{11 / 2},{ }^{2} \mathrm{~F}_{7 / 2},{ }^{4} \mathrm{~F}_{3 / 2},{ }^{4} \mathrm{~F}_{5 / 2},{ }^{2} \mathrm{H}_{9 / 2},{ }^{4} \mathrm{G}_{11 / 2},{ }^{2} \mathrm{G}_{7 / 2},{ }^{2} \mathrm{~K}_{15 / 2}$ et ${ }^{4} \mathrm{G}_{9 / 2}$ sont attribuées (tableau 3) [9].

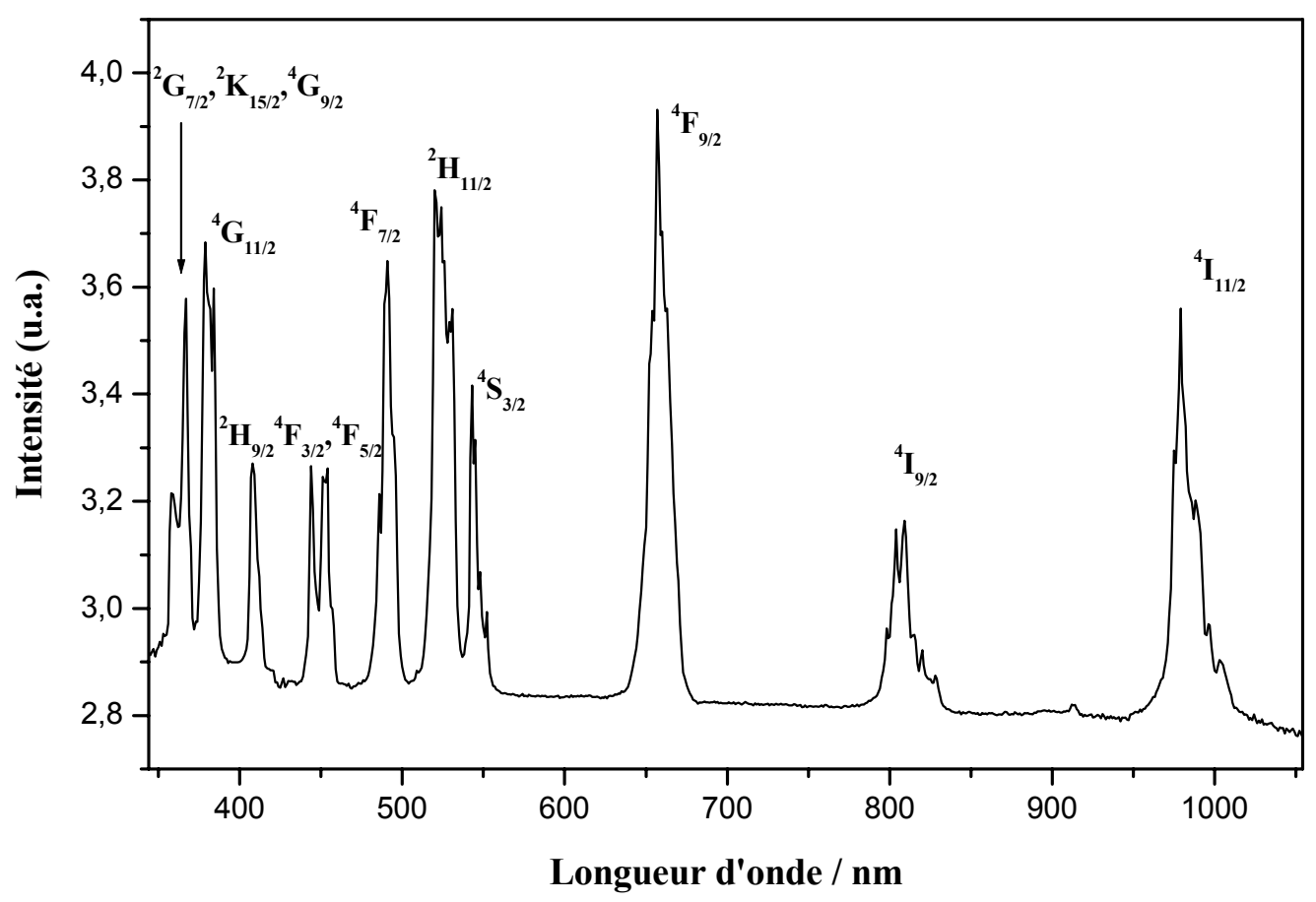

Figure 3. Spectre d'absorption à température ambiante de $\operatorname{RbEr}\left(\mathrm{PO}_{3}\right)_{4}$. 
Tableau 3. Longueurs d'onde correspondant aux transitions du niveau fondamental ${ }^{4} \mathrm{I}_{15 / 2}$ vers les niveaux excités de $\mathrm{Er}^{3+}$ dans $\mathrm{RbEr}\left(\mathrm{PO}_{3}\right)_{4}$.

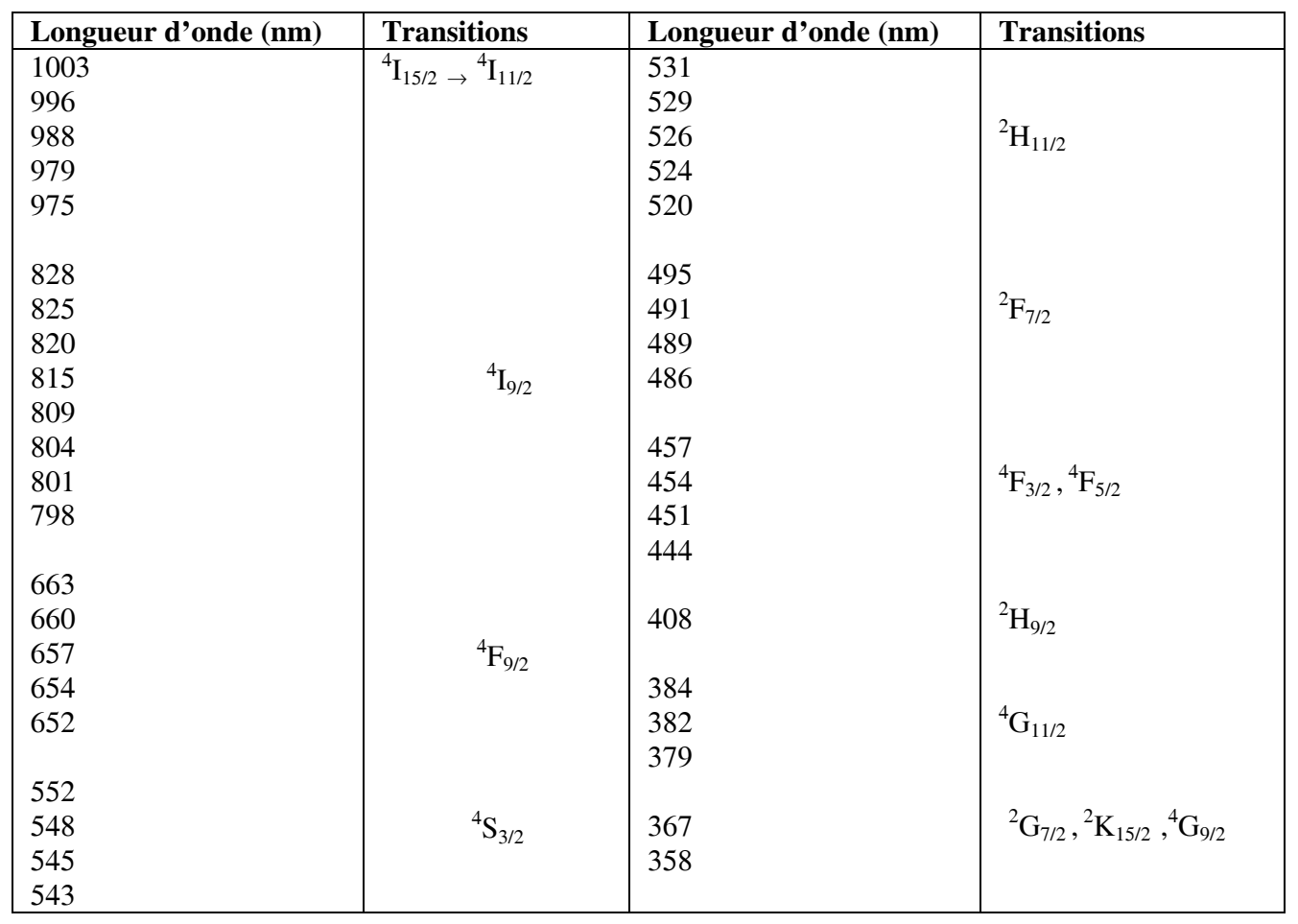

Le spectre d'absorption infrarouge de $\mathrm{RbEr}\left(\mathrm{PO}_{3}\right)_{4}$ (fig.4) montre que ce composé est un polyphosphate à chaînes infinies de tétraèdres $\mathrm{PO}_{4}$. Ceci est confirmé par la présence d'une large bande d'absorption entre 940 et $860 \mathrm{~cm}^{-1}$ correspondant à $v_{\text {as }}(\mathrm{POP})$, du multiplet $v_{\mathrm{s}}(\mathrm{POP})$ entre 680$810 \mathrm{~cm}^{-1}$ et du dédoublement des vibrations de déformation des tétraèdres $\mathrm{PO}_{4}$ entre 450 et $600 \mathrm{~cm}^{-1}$.

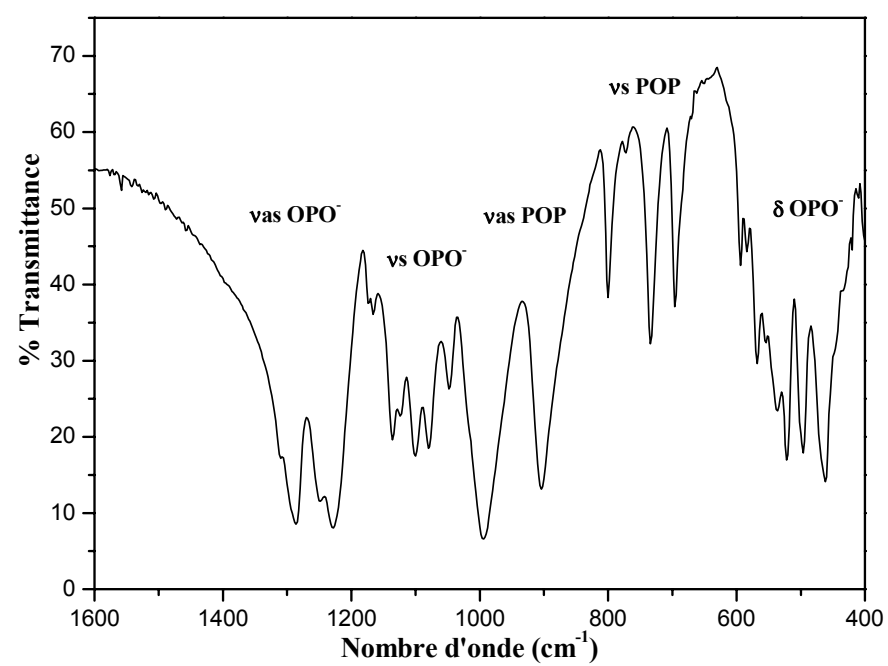

Figure 4. Spectre d'absorption infrarouge de $\operatorname{RbEr}\left(\mathrm{PO}_{3}\right)_{4}$. 


\section{Références}

[1] G. Blasse et B. C. Grabmaier, "Luminescent materials", Springer-Verlag (1994)

[2] M.J. Weber, "Handbook of Laser Wavelenghts", CRC Press (1999)

[3] W. Wolinski, M. Malinowski et R. Wolski, Proceedings of the $9^{\text {th }}$ International Congress, Laser 89 Optoelektronik (1990) 173

[4] A-F. Obatton, C. Parent, G. Le Flem, P. Thony, A. Brenier et G. Boulon, J. Alloy. Comp. 300-301 (2000) 123

[5] WinGX - version 1.63, L. J. Farrugia, J.Appl. Cryst. 32 (1999) 837

[6] SHELXS 97, G.M. Sheldrick, Program for Crystal Structure Determination, University of Göettingen, Germany, (1997)

[7] SHELXL 97, G.M. Sheldrick, Program for Crystal Structure Determination, University of Göettingen, Germany, (1997)

[8] K. K. Palkina, Izv. Akad. Nauk SSSR, Neorg. Mater.18(9) (1982) 1413

[9] W. Ryba-Romanoxski, Z. Mazurak, B. Jezowska-Trzebiatowska, D. Schultze, and Ch. Waligora, Phys. Stat. Sol. (a) 62 (1980) 75 